\title{
A Survey on Uyghur Ontology
}

\author{
Hankiz Yilahun ${ }^{1}$, Seyyare Imam $^{2}$ and Askar Hamdulla ${ }^{3}$ \\ ${ }^{1}$ Institute of Information Science and Engineering, Xinjiang University, China \\ ${ }^{2}$ College of politics and public administration, Xinjiang University, China \\ ${ }^{3}$ School of Software, Xinjiang University, Urumqi, China 830046, \\ Corresponding Author \\ hansumuruh@xju.edu.cn,sayyarim@163.com,askarhamdulla@gmail.com
}

\begin{abstract}
Ontology has become a hot research topic in the fields of artificial intelligence such as knowledge representation, knowledge engineering and natural language processing (NLP) etc. In this paper, according to the application requirements in the intelligent Uyghur information retrieval system, by giving the brief description about the ontology and its construction rules, methods, tools and descriptive languages, have conducted the contrastive analysis the current research status about the ontology in domestic and abroad, and then sum up some key issues in Uyghur ontology construction procedures and some early achievements. After all, the further research directions are also proposed in this paper.
\end{abstract}

Keywords: Ontology; Protégé; OWL; Uyghur

\section{Introduction}

Ontology is a concept of philosophy. In the philosophical sense, Ontology is concerned with the "existence", that is in essence, what exists in the world or what kind of entities existing in the world. So, in the philosophical field, Ontology is an objective description of the real existence of any field in the world [1]. With the development of various subjects, the application domain of Ontology is drawn from the initial philosophy to the computer, and has gradually expanded to the library information, artificial intelligence, and medical intelligence field and so on. And also, it has been widely attended and applied in the related fields. In computer artificial intelligence, the earliest definition of ontologies is from Neches, Fikes et al. [2]. They defined ontology as "ontology defines the basic terms and relations comprising the vocabulary of topic area, as well as the rules for combining terms and relations to define extensions to the vocabulary". After than, more and more people study Ontology, and gives a number of different definitions in the information system, knowledge system and other fields. One of the most famous and widely cited definitions is given by Gruber [3] in 1993, it is also a relatively simple definition — “ a specification of a conceptualization” . Intuitively, conceptualization is the relevant informal knowledge one can extract and generalize from experience, observation, or introspection. The specification is the encoding of this knowledge in representation language. Burst[4] gave another definition based on Gruber's in 1997, which is "It is an explicit formal specification of a shared conceptualization". Noy F. N. and Mc Guinness from Stanford University also proposed the new one in 2001 that is "ontology is a formal explicit description of concepts in a domain" [5]. In fact, there is no obvious difference between these definitions.

From the definition of the different researchers, the concept of ontology is defined as the term (vocabulary), relation, and conceptualization, and formal specification, domain knowledge, semantic, sharing and reasoning. Popularly speaking, Ontology is essentially a conceptual model that provides for the computer with the expression of the relations 
between concepts; Formally, Ontology is expressed as a vocabulary list, which aims to provide a clear expression of the relevant domain knowledge, and thus realize the semantic communication between human and computer. The ultimate goal of ontology is capturing knowledge in related fields, provide a common understanding and identify the common recognition of vocabulary. After that provide modeling tools for this field based on semantic and knowledge level from different formal model [6-7].

To the elements of the ontology, different researchers have different views but are similar, after summarizing can be obtained some parts follow as:

(1) Class or Concept: is a collection of concepts expressed by similar terms. As an example, " traffic" is a class, " railway, highway, waterway and airway" are sub class of the " traffic" class.

(2) Property: is used to describe the concepts in a class, and it has the function that restricting the concepts and instances. For example, the class "Xinjiang people" have properties like "nation" and "customs and habits" etc. where the type of "nation" is enumeration type, its scope include "Uyghur, Kazak, Kirgiz, Hui, Chinese" etc..

(3) Function: is a specific expression of the relation. The mapping relation in the function can reason another concept from one concept. For example, function Mother (x, y) express $y$ is x's mother, and function value is only one, but the value of inverse function Children () is not only one.

(4) Axiom: is the logic eternal truth that is not required to prove the truth, which is used for knowledge reasoning. For example, the two parallel lines never intersect.

(5) Instance: is a representative element, also called an individual. In the final analysis, the class is a class of the instance, and the instance is an instance of the class; the function is a function of the instance, and the instance is an instance of the function. Instance is the smallest object in Ontology, and has the properties of atomic and indivisible. If an instance can also be divided, then it is a class, not an instance. Instance can be operation in the function and the result must be another instance or class. Classes contain instances, and each instance has a property that does not belong to another instance, which is the only representation of a different instance. For example, "Urumqi" and "Karamay" is two different instances of class "city".

Since the ontology has different meanings in different fields, the Giaretta and Guarino of Stanford University proposed to represent the ontology research in Philosophy field by using Ontology (initial letter upper cased), and by using ontology (initial letter lower cased) represent the ontology system and basic theory study in artificial intelligence field ${ }^{[8]}$. In this paper, we will follow this rule in the use of the term "ontology".

\section{Classification and Construction Principle}

At present, according to different classification standards of the type of ontology there are different views. One of the most influential classification method is to divide the ontologies into four categories, that are top-level ontologies, domain ontologies, task

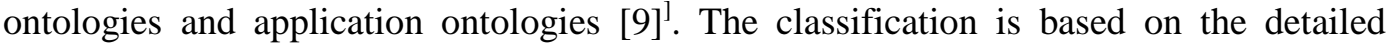
degree of ontologies and domain dependence. Among them: top-level ontologies is described as a general concept, usually not dependent on a particular area or specific problem. For example, Cyc, WordNet, SUMO, HowNet etc.; Domain ontologies is the ontology that describes the domain of a particular subject. It describes the main activities, the main related theories and principles in the field by the rich semantic relations between the terms in a domain. For example, GO (Gene Ontology), EO (Enterprise Ontology), BO (Biomedical Ontology) etc.; Task ontologies describes the relations between concepts in specific tasks or activities. For example, Dialogue Ontology, Sales Ontology, etc.; Application ontologies describes the relations between concepts that are dependent on specific areas and specific tasks at the same time. In these ontologies, it is focused at 
domain ontologies in domestic and foreign. The hierarchical relations between these ontologies are shown in figure 1[10].

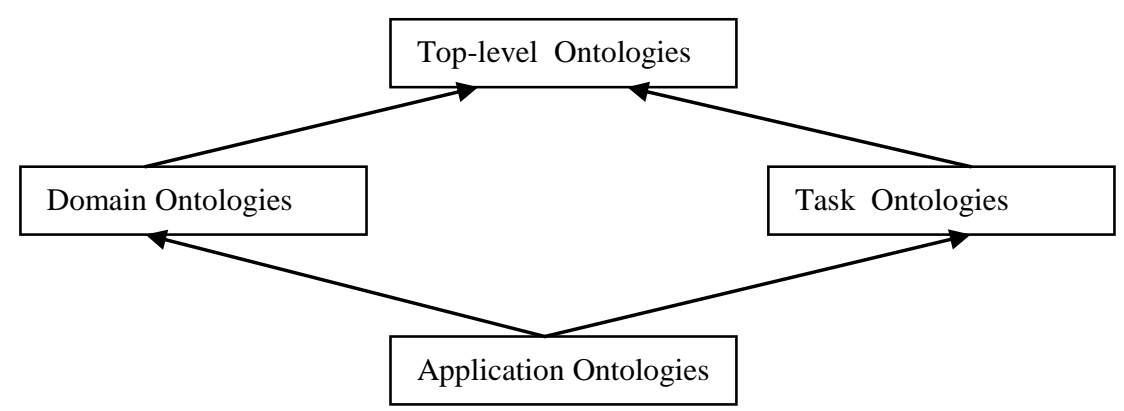

Figure 1. Ontology Classification and Hierarchy

The process of ontologies construction is very time-consuming and laborious work. It needs a set of perfect engineering system method to support. Specific domain ontologies also requires the participation of domain experts in order to guarantee that it accordance with quality requirements and application needs of users. Though the research of ontology is in full swing, there is still no unified guidance modeling method for the construction. The researchers all take a certain process to ontology modeling out of consideration for their own problems and specific project. In order to guide the people doing ontologies construction, researchers summed up some standard for guidance of ontologies modeling in research field. They combined with practice experience according to the specific projects, in which the most influential is ontologies modeling five rules of Gruber's [11] .

(1) Clarity and objectivity: This rule describes and defines these terms and relations between them with explicitly and objectively using natural language.

( 2 ) Completeness: The definition of concepts and properties should be as comprehensive and complete as possible.

(3) Consistency: It requires that the reasoning conclusion of the ontologies should be consistent with the meaning of the term itself and there is no contradiction before and after.

(4) Maximal monotony extendibility: It will not affect the existing content when you add terms and properties to a good ontology.

(5) Minimum promise: The constraint conditions for the modeling object should be as little as possible.

In the aspect of ontology construction, although there are not yet formed a set of standard unified guidance for modeling method, the application of the five rules still can be a good guide to the modeling process.

\section{Construction Method}

The main title (on the first page) should begin $13 / 16$ inches ( 7 picas) from the top edge of the page, centered, and in Times New Roman 14-point, boldface type. Capitalize the first letter of nouns, pronouns, verbs, adjectives, and adverbs; do not capitalize articles, coordinate conjunctions, or prepositions (unless the title begins with such a word). Please initially capitalize only the first word in other titles, including section titles and first, second, and third-order headings (for example, "Titles and headings" — as in these guidelines). Leave two blank lines after the title.

It is gradually realized that ontology can play an important role in many domains and tasks, which promotes the construction of ontologies in a certain extent. The methodology of ontologies construction is also in constant maturity. But there is still no such a method similar to the UML language in the software engineering to guide through the entire 
construction of ontology. Most existing methods are combined with a specific project. Although some experts have developed a variety of comprehensive methods to construct ontologies, such as IDEF-5, Skeleton method, TOVE, SENSUS, METHONTOLOGY, KACTUS, Circulating method, seven step methods etc, because of the domain, scope and specific project are all different, there is not a unified ontologies construction method yet.

The aim of creating ontologies is accessing domain knowledge in a general way. Ontologies provide a common understanding of a given field to realize knowledge sharing and reusing in different applications or organizations ${ }^{[11]}$. The function of ontologies is a kind of knowledge organization system, in the computer domain, information organization domain etc. However, construction of Knowledge Organization (description) system is a huge project. It is impossible to implement a general knowledge description system covering all fields of knowledge by part people's efforts alone. The practical and feasible method is starting from a specific field to construct a knowledge description system covering the domain. After the research found that the existing guiding ideology of the methodology and the construction process is not much difference. Therefore, in this paper the basic process of domain ontologies construction is presented as figure2. It is based on the existing methods of ontologies construction, then combining with specific areas of demand and characteristics.

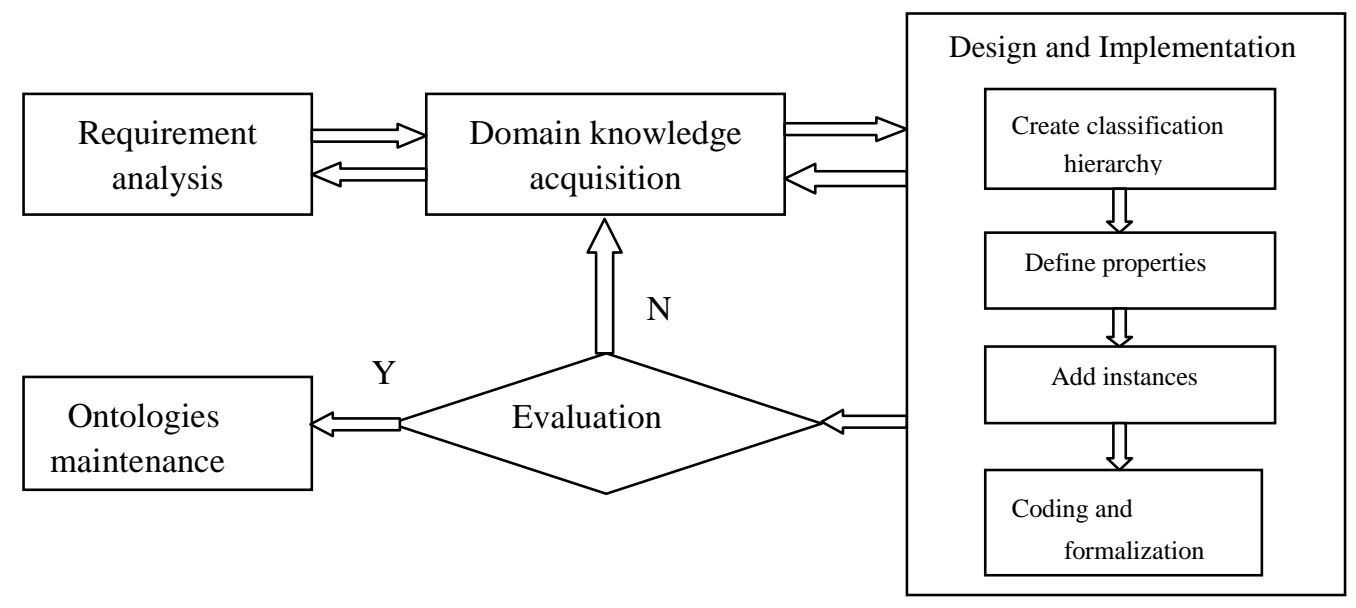

Figure 2. Domain Ontologies Construction Process

Now, let's explain every construction step as follow:

First, it is an important work to determine the main body of the field, scope, purpose, users and other basic information for ontologies construction, that is, the requirement analysis should be done well;

Second, ontologies construction is a large project, which needs the cooperation of domain experts. The domain knowledge acquisition and definition of the relations between concepts can only be completely understood by them;

Third, Ontologies design includes these following works that definition of concepts, objects, properties and relations. So it is a core link of ontologies construction. According to the result of the latest step we can list which is concepts, which is an object and which is a property, than determine which the relation between them is. There are four types of basic relations of domain ontologies in semantic, follow as table 1. However, in practical application, the relations between concepts are not limited to these four kinds. We can increase or decrease it according to actual situation. The relations are a subset of $\mathrm{N}$ dimensional Decare product: $\mathrm{C} 1 \times \mathrm{C} 2 \times \cdots \times \mathrm{Cn}[12]$. Gene Ontology, in bioinformatics, although include the easy relation-isPartOf, there are also a tremendous impact in the field. So it is flexible definition in practical ontologies construction. For example, the 
relations between the concepts, which human, animal and plant, we can choose "eat" and "eated".

Fourth, the preparation is completed after designing the class, property, instance and relations. It is need to implement the ontologies, that is, choose a suitable programming language and ontology building tools, to make the computer understand the structure of the organizations and relations.

Fifth, test and evaluate. It is need to test and evaluate such problems after ontologies construction, that is whether the ontology formulization meet demand or not, whether domain knowledge is considered adequately or not, whether the terms are clearly defined, whether the concepts and its relations are completely or not and so on.

Sixth: ontology maintenance. A series procedures of error correction, improvements and application maintenance work etc. After that one can realize the continuous improvement and evolution of ontologies.

Table 1. Types and Descriptions of Basic Relations

\begin{tabular}{|c|l|l|}
\hline name & \multicolumn{1}{|c|}{ description } & example \\
\hline isPartof & The relation between part and whole classes. & $\begin{array}{l}\text { "CPU"isPartOf" } \\
\text { Laptop" }\end{array}$ \\
\hline isKindof & $\begin{array}{l}\text { The relation between parent and child classes, Also be called } \\
\text { abstract and specific relation. It is important for the realization } \\
\text { of semantic word expansion. }\end{array}$ & $\begin{array}{l}\text { "Laptop"isKind } \\
\text { Of "Computer" }\end{array}$ \\
\hline isInstanceof & $\begin{array}{l}\text { The relation between concepts and its instances. It is similar to } \\
\text { the relation between objects and classes in object-oriented. }\end{array}$ & $\begin{array}{l}\text { "Urumqi'isInsta } \\
\text { nceOf "City" }\end{array}$ \\
\hline isAttributeof & $\begin{array}{l}\text { Representing a concept is property of another concept. There } \\
\text { are two property in ontologies, that are object property and } \\
\text { data property. }\end{array}$ & $\begin{array}{l}\text { "Brand" } \\
\text { isAttributeOf"La } \\
\text { ptop" }\end{array}$ \\
\hline
\end{tabular}

\section{Tools and Description Language}

Ontology construction is a very complicated system engineering, which needs the right development guidance and suitable tools. A good editing software can provide input ontology, consistency checking, visualization, query, reasoning and other functions. At present, there are many mature ontology development platform software available for selection: one kind of tools is based on a specific ontology description language. Representative tools are Ontolingua, Ontosaurus and Webonto. In which Ontolingua is based on Ontolingua language, Ontosaurus is based on LOOM language, and Webonto is based on OCML language. Another kind of tools can support for multiple languages. They are OilEd, OntoEdit, webODE and Protégé [13] etc..

With the increasing application of Ontology in the computer field, researchers have carried out a large number of projects, and have developed many kinds of construction tools. According to incomplete statistics, at present there are more than 60 kinds of ontology construction tools, where higher visibility and more widely used tools are more than10 kinds. And in which had been applied relative widely is Protégé. Protégé was developed by the Stanford Center for Biomedical Informatics Research at the Stanford University School of Medicine. It is supported by a strong community of academic, government, and corporate users, who use Protégé to build Knowledge-based solutions in areas as diverse as biomedicine, e-commerce, and organizational modeling. Protégé can be widely used in the research and application of ontology, because of its huge advantage compared with other tools. It can be expressed in the following aspects: 
(1) Protégé is a free, open-source platform that provides a growing user community with a suite of tools to construct domain models and knowledge-based applications with ontologies.

(2) Protégé support for the multi lingual ontology, especially for the construction of Chinese and Uyghur ontology, follow as Figure 3.

(3) Protégé fully supports the latest OWL 2 Web Ontology Language and RDF specifications from the World Wide Web Consortium.

(4) Protégé supports database storage, access the database via JDBC and JDBCODBC bridge.

( 5 ) Protégé's is based on Java, is extensible, and provides a plug-and-play environment that makes it a flexible base for rapid prototyping and application development.

(6) Support reasoning: One hand Protégé carry with its own logic reasoners like FaCT++ HermiT, etc., on the other hand developer can add another one like Pellet.

Protégé also provides an ontology mechanism for cooperative development, allows to annotate for components and changes, supports for queries and filtering based on different standards of user comments. At present, Protégé provides two models for the cooperative development [14]. One is multi user mode: It allows multiple clients to simultaneously edit ontologies on the Protégé. Any change made by a user is immediately visible to other users. This mode can be seen as a client / server mode or synchronous mode, is the first choice for multi-user development. For example, WebProtege is Another independent ontology, also called as continues mode: It allows multiple users to access the same ontology continuously. This ontology can be stored in shared network drivers, all users can access the same project.

This paper uses the OWL-based Protégé4.3 tool based on the above advantages.

There are a lot of ontology description languages, such as XML, XML Schema, RDF, RDF Schema. In which OWL(Web Ontology Language) is based on $\mathrm{RDF}(\mathrm{S})$, uses $\mathrm{RDF} / \mathrm{XML}$ 's grammar. So OWL's meaning more widely than RDF's, it is an expansion of $\mathrm{RDF}^{[14]}$. OWL can be used to define the meaning of the term and the relationship between them, such relation between the terms is called as ontology. OWL has added more vocabulary for the class and property description, compared with XML, XML Schema and RDF Schema. For example, disjoint with, equivalent, symmetric, enumerate type and so on. Because of the advantages of OWL, we choose OWL language to describe Uyghur Ontology. The following is a part of the code described in OWL.

Class description:

<owl:Class rdf:about="\#乡゙ ئاق ره ">

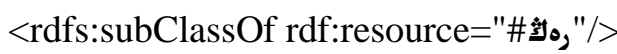

$</$ owl:Class>

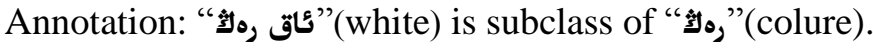

Relation description:

<owl:ObjectProperty rdf:about=" \#isPartOf"> <rdf:type rdf:resource="\&owl;TransitiveProperty"/>

$</$ owl:ObjectProperty>

Annotation:"isPartOf" has Transitive Property.

Instance description:

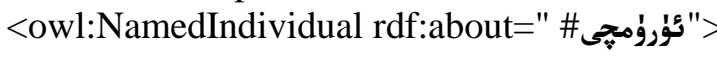

$<$ rdf:type>

<owl:Restriction>

<owl:onProperty rdf:resource=" isInstanceOf"/> 


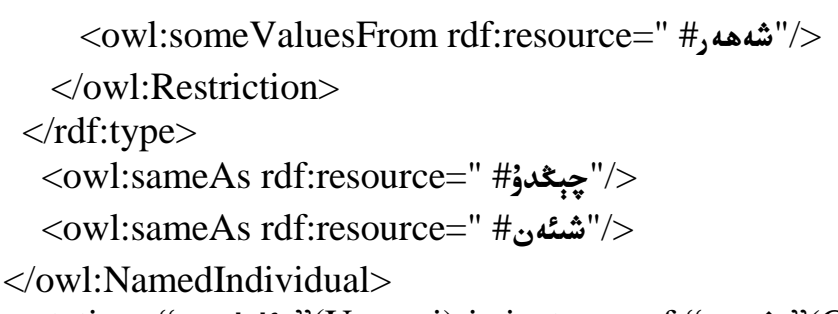

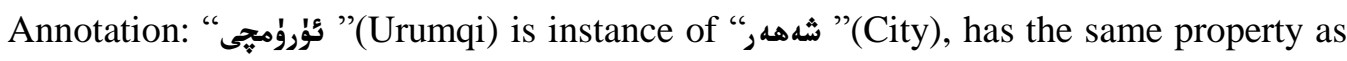

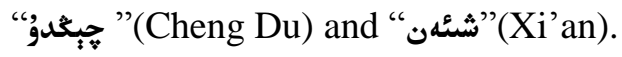

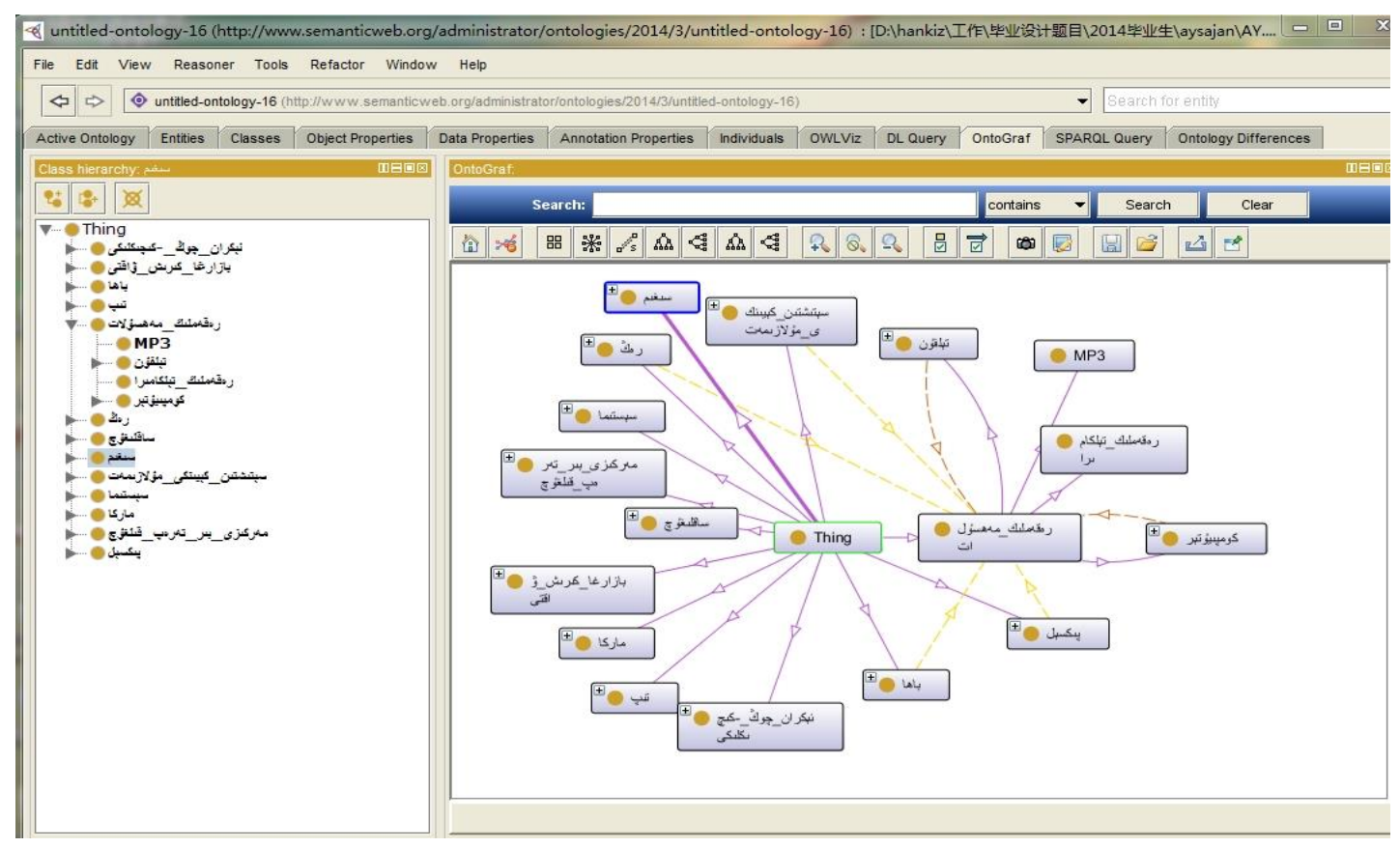

Figure 3. View Interface of Uyghur Ontology in Protégé4.3

\section{Research Status in Domestic and Foreign}

The second and following pages should begin 1.0 inch $(2.54 \mathrm{~cm})$ from the top edge. On all pages, the bottom margin should be 1-3/16 inches $(2.86 \mathrm{~cm})$ from the bottom edge of the page for $8.5 \times 11$-inch paper; for A4 paper, approximately 1-5/8 inches $(4.13 \mathrm{~cm})$ from the bottom edge of the page.

There are famous ontologies in domestic and foreign in current such as VerbNet, FrameNet, HowNet and WordNet, and they are all belong to the semantic dictionary. They extract semantic information from words and syntax, and present them in a net form, comparing with that traditional alphabetical sequence organization of lexical dictionary, these ontologies starting more from the concept of vocabulary, focus on the same, similar meaning or with a certain degree of relevance vocabularies. To understand the information contained in natural language by the computer, it will achieve semantic analysis and understanding use semantic resources [15].So we call it lexical ontology.

WordNet (WN)(http://wordnet.princeton.edu/) is a large lexical database of English. Nouns, verbs, adjectives and adverbs are grouped into sets of cognitive synonyms (synsets), each expressing a distinct concept. Synsets are interlinked by means of conceptual-semantic and lexical relations. The resulting network of meaningfully related words and concepts can be navigated with the browser. WN is also freely and publicly available for download. WN 's structure makes it a useful tool for computational linguistics and natural language processing. The most recent Windows version of WN Net is 2.1, released in March 2005. Version 3.0 for Unix/Linux/Solaris/etc. was released in 
December, 2006. Version 3.1 is currently available only online. WordNet3.0 include about 155287 words, where name is 146312 , verb is 25047 , adjective is 30002 , adverb is 5580, and all synsets is about 117659[14-15]. As an online dictionary, WordNet provides a good user interface, which can provide different computer platforms.

VerbNet (VN) (http://verbs.colorado.edu/) (Kipper-Schuler 2006, University of Pennsylvania USA) is the largest on-line verb lexicon currently available for English. It is a hierarchical domain-independent, broad-coverage verb lexicon with mappings to other lexical resources such as WordNet and FrameNet. There are 5247 verb senses distributed throughout 274 first-level classes using 36 Syntactic restrictions and 23 Thematic roles in currently version. At the level of syntactic and semantic description, VN used 357 syntactic frame and 94 Semantic predicates [16].

FrameNet (FN)(http://framenet. icsi.berkeley.edu/)project is building a lexical database of English that is both human- and machine-readable, based on annotating examples of how words are used in actual texts. For students and teachers of linguistics it serves as a valence dictionary, with uniquely detailed evidence for the combinatorial properties of a core set of the English vocabulary. The project has been in operation at the International Computer Science Institute in Berkeley since 1997, supported primarily by the National Science Foundation, and the data is freely available for download. The most recent version of $\mathrm{FN}$ is 1.3, and it has 1094semantic frame including 9382 frame units and 12132lexical units, where over than 6100 vocabulary has been annotated completely as well as over than 135000 example sentences[17-18]. However, the relations between semantic types in FN is single, only is sub class relations, lack of Axiom, so can only provide a shallow level of classification, reveal the shallow semantics.

HowNet (HN)(http://www.keenage.com/html/e_index.html) is an on-line commonsense knowledge base unveiling inter-conceptual relations and inter-attribute relations of concepts as connoting in lexicons of the Chinese and their English equivalents, which developed by Prof. Zhendong Dong at Chinese Academy of Sciences. The knowledge system of the computer department of University of Texas research group has listed HN as one of the ontology projects . Description of concepts in $\mathrm{HN}$ is an attempt to present the inter-relation between concepts and that between their attributes. At the same time, it also contains the English concepts and relations corresponding to Chinese. Where the Chinese vocabulary is about 50220 in the latest 2.0 version, where nouns, verbs, adjectives are 26037, 16657, 9768 respectively; The English vocabulary is about 55422, where nouns, verbs, adjectives are 28876, 16706, 10716 respectively [14-15]. Mr. Zhendong Dong groups establishes the knowledge network system which describes the object with all kinds of concepts via the top-down construction method based on the four hundred million words in Chinese corpus,

To the minority natural language processing in domestic, the knowledge acquisition technology is at the basic research stage. So there are not many studies on the ontology of the national language. Xiaobing Zhao et al.[19] has researched about multi language Ontology knowledge base, Lirong Qiu et al. [20] has presented about the method of ontology construction in Tibetan based on hyponymy relations. Qing Liye [21] has studied about semi auto expansion method for Mongolian ontologies. In addition, there are not any other relevant research results in other minority languages.

\section{Problems and Future Work}

Uyghur language is a mother language of the main ethnic minority (Uyghur) in Xinjiang and the surrounding areas. It is an adhesive language in morphological structure, and belongs to the Altai Turkic languages. There are vast and numerous classical literature, historical writings and translations in Uyghur language. Whether Uyghur language are as the main carrier of national culture heritage or as the main tool of spreading the knowledge of science and technology culture now, it is inestimable that the 
unique human culture value and the tremendous role in Xinjiang and its surrounding areas[22].

It is have been more than 20 years on the information processing technology of Uyghur studies, although the development has been made in all aspects and many achievements have been got, still can't keep up with the developing speed of the information age with the internet as the main body. If Uyghur language once cannot enter the information age, it will lose the basic function and roll of culture carrier, than will be mercilessly abandoned by this age. Therefore, Uyghur information processing is directly related to text fate, its significance is self-evident.

In recent years, the research on ontology theory and application are developing rapidly in domestic and foreign. Whether it is a theoretical research, empirical research, or the realization and application of technical means are in the ascendant, however, there are not yet related research and report about Uyghur language. Therefore, it is very necessary to collect and standardize about multiple domains knowledge, than construct Uyghur ontology. Its implementation will provide semantic resource support for Uyghur information retrieval, data mining, automatic summary generation, text clustering, semantic classification and related fields of intelligent information processing of semantic resources.

According to the above discussion of the ontology construction method as shown in Figure 2, so far this paper has constructed two domain ontologies, which information science (ISO) and mathematic (MO), via integrate the advantages and disadvantages of each method and using Protégé4.3. Where ISO contains 14 super classes, 899 subclasses, 100 individuals (instances), 60 Properties; MO contains 6 super classes, 92 sub classes, 885 individuals (instances), 6 Properties.

\subsection{Problems}

Currently, due to the method of ontology building is not a unified and standard; there are different ontology modelings by different experts, who may describe the knowledge in the field at different views or levels even in the same field. The main problems are summarized as follows:

(1) Insufficient demand: When the domain ontology is constructed, if the specific requirements of a certain domain are considered not adequately and the description is not clear, than it lead the possibility of ontology construction without planning and the need to redesign.

(2) Ontology heterogeneity: The same concept is different meaning in different ontologies. For example, concept A may be representing a man in Ontology1; however, it may be a company in Ontology2. Example again, the temperature may be expressed by degrees Celsius in Ontology1, but it may be degrees Fahrenheit in Ontology2. In addition, the relation between concept A and B may be the property_isPartOf in Ontology1, but it may be the property_isKindOf in Ontology2. There are also heterogeneity problems in our ontologies. For example, "tree" is subclass of "graph theory" in MO, however, it is sub class of "data structure" in ISO.

(3) Sharing and reusing: It is need to Ontology integration in different languages ontologies when reusing and sharing knowledge. However, due to the different description language of concepts, it is not easy to realize the merging, mapping and alignment of ontologies.

\subsection{Future Work}

With the trend of the internet information resources became semantic and multilingual, it is urgent need to apply the method and techniques of information semantic processing and multi language processing. Currently, there are two important trends in the 
development of ontology construction, one is domain based and another is multilingual [23]. Many international organizations, such as FAO, WHO, are all urgent need to implement machine translation and cross language information retrieval etc. based on multilingual ontology.

The foundation of ontology application is ontology construction. The traditional manual construction method of the system can guarantee the quality, but it takes time and effort. Ontology learning is an important method to solve the construction. It gets the expected ontology automatically (or semi-automatically) from existing data sources via uses the techniques, such as statistical, machine learning, NLP, automatic (semiautomatic) to obtain the desired data from the existing data sources. Therefore, one of future work of the paper is further studies on automatic extraction of the relations between concepts and implementation of automatic construction through learning ontology.

Another future work is extending of Uyghur ontology to Uyghur- Kazak-Kirgiz multilingual ontology. Because Xinjiang is a minority autonomous region and the main ethnics are Uyghur, Kazak, Kirgiz, the total population of the three ethnic groups accounted for more than half of the Xinjiang population. Therefore, it is very necessary to do information processing of Kazakh and Kirgiz language in addition Uyghur.

In brief, the situations considered in this paper about Uyghur ontology construction are relatively simple, the concepts and relations are comparatively small, lack of constraints on the relations, than the domain ontology is not complete. So it is need to further improve the research according to the needs of the follow-up application demand.

\section{Acknowledgements}

This work was supported by the National Social Science Foundation of China (13BYY062), Innovation Program for Excellent Ph.D. Candidates of Xinjiang University (XJUBSCX-2013011), and Natural Science Foundation of Xinjiang University (XY110103).

\section{References}

[1] Y. P. Huo, "Research On Key Technologies Of Agricultural Knowledge Management Based On Ontology", Agricultural Science and Technology Press, (2009); Beijing, China.

[2] T. R. Gruber, "A Translation Approach to Portable Ontology Specifications", Knowledge Acquisition, vol. 5, (1993), pp. 199-220.

[3] C. R. Huang, N. Calzolari and A. Gangemi, "Ontology And The Lexicon -- A Natural Language Processing Perspective", Peking University Press, (2014); Beijing.

[4] W. N. Borst, "Construction of Engineering Ontologies for Knowledge Sharing and Reuse", University of Twente, Enschede, (1997).

[5] M. Bahatt, W. Rahayu and G. Sterling, "Synthetic Environment Representation Semantic Using the Web Ontology Language", Lecture Notes in Computer Science, (2005), pp. 3578:9-16.

[6] N. Guarino, "Formal Ontology and Information System", Trento: IOS Press, (1998), pp. 6-8.

[7] H. Wang, S. L and H. B. Zhu, "Research on key issues of national basic geographic information ontology", Science Press, (2011).

[8] N. Guarino and P. Giaretta, "Ontologies and Knowledge Bases: Towards a Termino-logical Clarification", Towards Very Large Knowledge Bases. N. J. I. Mars. Ed. IOS Press, (1995).

[9] X. P. Wei, "Construction And Application Of Hepatitis Virus Protein Domain Ontology", Shanghai Jiao Tong University, (2013), pp. 2-3.

[10] Z. H. Deng, Y. L. Zhao, C. L. Li and P. Tang, "Multi-Lingual Thesauri-Ontology", Wuhan University Press, (2011).

[11] Z. Xu, Y. Y. Jiang and Z. Y. Li, "Construction and Application of Ontology in Tranc Surveillance Video Systems", Journal of Shanghai University(Natural Science), vol. 20, no. 5, (2014), pp. 658-668.

[12] H. X. Liu, "Research on Construction and Retrieval of Diabetes Ontology", Jilin University, (2014), pp. $16-17$.

[13] X. Y. Wang and B. Xiao, "Query Expansion Method Based on Ontology and Local Context Analysis", Computer Engineering, vol. 7, (2012), pp. 57-59.

[14] G. Gorkem and U. M. Osman, "A method for ontology-based semantic relatedness measurement", Turkish Journal of Electrical Engineering \& Computer Sciences, vol. 21, (2013), pp. 420-438. 
[15] Z. Z. Jia, Y. F. Tai, Y. L. Liu and D. Guo, "Research on the knowledge ontology of Chinese FrameNet", Science Press, (2012).

[16] R. J. Bai, X. M. Yu and X. Y. Wang, "The Comparative Analysis of Major Domestic and Foreign Ontology Library", Journal of Modern library and information technology, vol. 1, (2011), pp. 3-13.

[17] J. Z. Jia and Y. H. Chen, "Construction of Ontology in Chinese Language Framework Network", Chinese Library Journal, vol. 2, (2007), pp. 56-64.

[18] R. Li, "Research on Frame Semantic Structure Analysis Technology for Chinese Sentences", Shanxi University, (2012), pp. 11-13.

[19] X. B. Zhao, L. R. Qiu and T. J. Zhao, "Construction Technology Of Ontology Knowledge Base In Multiple Minority Language", J. Of Chinese Information Processing, vol. 25, no. 4, (2011), pp. 71-74.

[20] L. R. Qiu, Y. Weng and X. B. Zhao, "Acquisition Method of Hyponymy Concepts Based on Patterns in Tibetan Semantic Ontology", Journal Of Chinese Information Processing, vol. 25, no. 4, (2011), pp. 45 49.

[21] Y. Q. Li, L. R. Qiu and X. B. Zhao, "Semantic Expansion Method of Mongolian Ontology in the Perspective of Internet", Journal of Minzu University of China (Philosophy and Social Sciences), vol. 42, no. 1, (2015), pp. 142-145.

[22] T. Ibrahim and Y. Baoshe, "A Survey on Minority Language Information Processing Research and Application in Xinjiang", J. Of Chinese Information Processing, vol. 25, no. 6, (2011), pp. 149-156.

[23] L. Zhao, H. Ren and J. Wan, "Automatic Ontology Construction Based on Clustering Nucleus", Wuhan University Journal of Natural Sciences, vol. 20, no. 2, (2015), pp. 129-133.
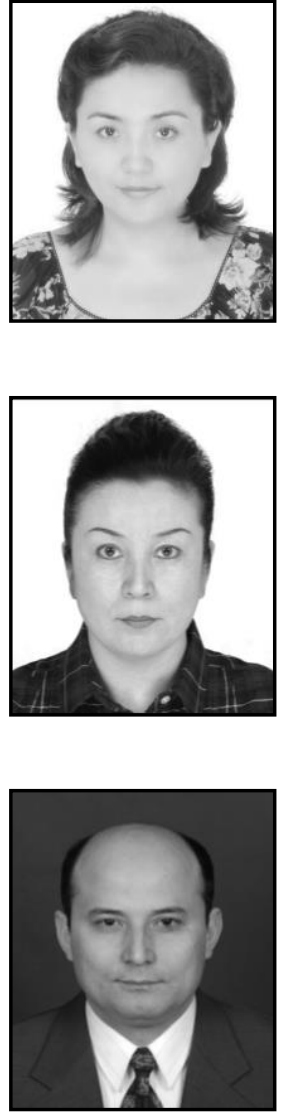

\section{Authors}

Hankiz Yilahun, she has received his B.E. and M.S. degree in Computer Science and Technology from Xinjiang University and Beijing University of Technology, China, in 2002 and 2009, respectively. Currently, he is a $\mathrm{PhD}$ candidate in Computer Applications in Xinjiang University, and working as a teacher at Mathematics and System Science, Xinjiang University, China. His research interests include Uyghur ontology and its applications.

Seyyare Imam, she received her B.E. and M.S. degree in politics and philosophy, from Xinjiang University and Xinjiang Normal University of China, in 1995 and 2008, respectively. Since 1996, she has been working as a teacher in politics and public administration, Xinjiang University. Her research interests include social work, social language, and related issues about Uyghur ontology construction etc..

Askar Hamdulla, he received B.E. in 1996, M.E. in 1999, and Ph.D. in 2003, all in Information Science and Engineering, from University of Electronic Science and Technology of China. In 2010, he was a visiting scholar at Center for Signal and Image Processing, Georgia Institute of Technology, GA, USA. Currently, he is a professor in the School of Software Engineering, Xinjiang University. He has published more than 140 technical papers on speech synthesis, natural language processing and image processing. $\mathrm{He}$ is a senior member of CCF and an affiliate member of IEEE. 
International Journal of Database Theory and Application Vol.8, No.4 (2015) 